\title{
Accurate and fast computations with positive extended Schoenmakers-Coffey matrices
}

\author{
J. Delgado ${ }^{3 *}$, G. Peña ${ }^{1}$, J. M. Peña ${ }^{2}$ \\ Departamento de Matemática Aplicada, Universidad de Zaragoza, Escuela Universitaria Politécnica de Teruel, \\ E-44071 Teruel, Spain.
}

\begin{abstract}
SUMMARY
Schoenmakers-Coffey matrices are correlation matrices with important financial applications. Several characterizations of positive extended Schoenmakers-Coffey matrices are presented. This paper provides an accurate and fast method to obtain the bidiagonal decomposition of the conversion of these matrices, which in turn can be used to compute to high relative accuracy the eigenvalues and inverses of positive extended Schoenmakers-Coffey matrices. Numerical examples are included. Copyright (c) 2015 John Wiley \& Sons, Ltd.

Received ...
\end{abstract}

KEY WORDS: Accurate computations; Bidiagonal factorization; Total Positivity; Green matrix; Schoenmakers-Coffey matrix; Lehmer matrix

\section{INTRODUCTION}

Correlation models play a key role in many financial applications (cf. [18, 24, 27]). In particular, to the fields of derivatives pricing and to risk management. A very successful correlation parametrization has been provided by Schoenmakers and Coffey in [29]. A slight generalization of the Schoenmakers-Coffey correlation matrix was provided in [18] with the matrices that we call extended Schoenmakers-Coffey matrices, which are positive definite symmetric matrices. In [18] it is also shown that extended Schoenmakers-Coffey matrices present important applications in financial mathematics. Here we restrict our study to positive extended Schoenmakers-Coffey matrices in order to apply results of Total Positivity theory. In Section 2, we present several characterizations of positive extended Schoenmakers-Coffey matrices. These characterizations use concepts and tools coming from the Total Positivity field (see $[1,5,9,14,23]$ ), which is an interdisciplinary subject related to variation diminishing transformations and with applications to many other subjects in addition to Economics, such as Statistics, Approximation Theory, Computer Aided Geometric Design or Mechanics.

As recalled in Section 2, an $n \times n$ positive extended Schoenmakers-Coffey matrix $A$ belongs to the class of oscillatory matrices, which a subclass of nonsingular totally positive matrices, and so $A$ has $n$ positive and different eigenvalues and the number of variation of signs in the consecutive components of the eigenvectors corresponding to their $k$ th greater eigenvalue is $k-1, k=1, \ldots, n$. The application of this last property to the first three eigenvectors gives important information in financial problems (see [18, 25, 26, 27]). In fact, these shape properties of the first three eigenvectors of the correlation matrices for forward rates in a relevant class of models are known as shift

\footnotetext{
${ }^{*}$ Correspondence to: Departamento de Matemática Aplicada, Universidad de Zaragoza, Escuela Universitaria Politécnica de Teruel, E-44071 Teruel, Spain. Email: (jorgedel@unizar.es). 
(or level), slope and curvature (see [18, 25, 26, 27]). In fact, if the first three eigenvectors have respectively zero, one or two sign changes, then we say that we observe shift (or level), slope and curvature. Observe that the fact that the first eigenvector (which is known as the Perron eigenvector and corresponds to the largest eigenvalue) has zero changes of sign holds for any nonnegative matrix by the well known Perron-Frobenius theorem.

Let us mention that Lehmer matrices (see [12], [13]) belong to the class of positive extended Schoenmakers-Coffey matrices, as shown in Section 2, and so the results of this paper can be applied to Lehmer matrices.

Let us recall that an algorithm can be computed to high relative accuracy (HRA) when it only uses products, quotients, additions of numbers with the same sign or subtractions of initial data (cf. [8]). Among the classes of matrices for which algorithms to HRA have been constructed, we can mention some subclasses of nonsingular totally positive matrices (see [19, 20, 6, 7]). As shown in [16], for a nonsingular TP matrix $A$, the adequate parametrization to obtain computations to HRA is its bidiagonal factorization $\mathcal{B D}(A)$. Given a matrix $A=\left(a_{i j}\right)_{1 \leq i, j \leq n}$, the conversion matrix of $A$ is the matrix $A^{\#}:=\left(a_{n+1-i, n+1-j}\right)_{1 \leq i, j \leq n}$. In Section 3 we provide a method of $\mathcal{O}(n)$ elementary operations to obtain the $\mathcal{B D}\left(A^{\#}\right)$, where $A$ is an $n \times n$ positive extended Schoenmakers-Coffey matrix. Given this bidiagonal factorization with HRA, the eigenvalues and the inverses of the matrix $A$ can be also computed with HRA following the procedure presented in Section 3 and using the tools of [16].

Since positive extended Schoenmakers-Coffey matrices are symmetric, their eigenvalues coincide with their singular values. Besides, let us also recall the importance of Principal Component Analysis in many financial models of interest rate dynamics (see [18, 24, 25, 26, 27]). In these models the eigenvalues are the variances of the principal components. In fact, the estimation of the eigenvalues of the correlation matrices of forward rates is an important problem in this field (see $[24,25])$. Section 4 includes numerical examples illustrating the accuracy provided by our method joint with Koev's algorithms of [17] in order to compute eigenvalues, inverses or the solution of some linear systems.

\section{BASIC DEFINITIONS AND CHARACTERIZATIONS}

Let us introduce some basic notations. Let $A=\left(a_{i j}\right)_{1 \leq i, j \leq n}$ be a square $n \times n$ real matrix. We introduce a notation for the square submatrices of $A$. Given a positive integer $k \leq n, Q_{k, n}$ will denote the totality of strictly increasing sequences of $k$ natural numbers less than or equal to $n$ :

$$
\alpha=\left(\alpha_{i}\right)_{i=1}^{k} \in Q_{k, n} \quad \text { if } \quad(1 \leq) \alpha_{1}<\alpha_{2}<\ldots<\alpha_{k}(\leq n) .
$$

Given $\alpha, \beta \in Q_{k, n}$, then $A[\alpha \mid \beta]$ is by definition the $k \times k$ submatrix of $A$ containing rows numbered by $\alpha$ and columns numbered by $\beta$. When $\alpha=\beta$ the principal submatrix $A[\alpha \mid \alpha]$ is simply denoted by $A[\alpha]$ and $\operatorname{det} A[\alpha]$ is the corresponding principal minor. If $\alpha=(1,2, \ldots, k)$, then $A[\alpha]$ is called the leading principal submatrix of $A$ of order $k$ and $\operatorname{det} A[\alpha]$ is called the leading principal minor of $A$ of order $k$.

A symmetric matrix $A$ such that $x^{T} A x>0$ for all nonzero vector $x \in \mathbf{R}^{n}$ is called positive definite. It is well known that positive definite symmetric matrices are characterized by the property that all their eigenvalues are positive and also by the property that all their leading principal minors are positive. We say that a matrix $A=\left(a_{i j}\right)_{1 \leq i, j \leq n}$ has unit diagonal if all its diagonal entries satisfy $a_{i i}=1$ for all $i=1, \ldots, n$. The superdiagonal entries of $A$ are given by the entries $a_{i, i+1}$ for all $i=1, \ldots, n-1$ and the subdiagonal entries are given by the entries $a_{i+1, i}$ for all $i=1, \ldots, n-1$. $A$ is a tridiagonal matrix if all its entries are null up to its diagonal, superdiagonal and subdiagonal entries, that is, $a_{i j}=0$ whenever $|i-j|>1$.

As in [18], we shall consider by simplicity that a covariance matrix is a positive definite symmetric matrix. If, in addition, it has unit diagonal, then it is a correlation matrix. Since a positive definite matrix has all its principal minors positive, a correlation matrix $R=\left(\rho_{i j}\right)_{1 \leq i, j \leq n}$ satisfies that, for any $i \neq j, 0<\operatorname{det} R[i, j]=1-\rho_{i j}^{2}$ and so $\left|\rho_{i j}\right|<1$. Empirical evidence (cf. 
$[24,25,26,27,28,29])$ suggests that correlations $\rho_{i j}$ of interest rates satisfy the following properties:

P0 $\rho_{i j}>0$ for all $i, j$,

P1 $\left\{\rho_{i j}\right\}$ is strictly increasing in $i$ and strictly decreasing in $j$ when $i<j$.

By symmetry of $R, \mathrm{P} 1$ implies a corresponding property when $i>j$. Property $\mathrm{P} 0$ implies that interest rates at different maturities are positively correlated and P1 implies that the correlation coefficients decrease when the distance between the indices increases. The strict increase of superdiagonal entries is another property suggested by the empirical evidence and that will be also considered:

P2 $\left\{\rho_{i, i+1}\right\}$ is strictly increasing in $i$, which implies by symmetry of $R$ a corresponding property for the subdiagonal entries.

A Green matrix $G=\left(g_{i j}\right)_{1 \leq i, j \leq n}$ is a symmetric matrix such that, for each $i \leq j, g_{i j}:=u_{i} v_{j}$, where all $u_{i}, v_{j}$ are real numbers different from zero. We say that $G$ is associated to $\left\{u_{i}\right\}_{1 \leq i \leq n}$ and $\left\{v_{i}\right\}_{1 \leq i \leq n}$.

The following result, which corresponds to (6) of [28] (see also [4, 3, 9, 14]) provides the inverse of a Green matrix.

\section{Theorem 1}

Let $G$ be the $n \times n$ Green matrix corresponding to the sequences $\left(u_{i}\right)_{1 \leq i \leq n}$ and $\left(v_{i}\right)_{1 \leq i \leq n}$. Then, its inverse $C=G^{-1}=\left(c_{i j}\right)_{1 \leq i, j \leq n}$ is the tridiagonal matrix given by:

$$
\begin{aligned}
c_{11} & =\frac{u_{2}}{u_{1}\left(u_{2} v_{1}-u_{1} v_{2}\right)}, \\
c_{i i} & =\frac{u_{i+1} v_{i-1}-u_{i-1} v_{i+1}}{\left(u_{i} v_{i-1}-u_{i-1} v_{i}\right)\left(u_{i+1} v_{i}-u_{i} v_{i+1}\right)}, \quad i=2, \ldots, n-1, \\
c_{n n} & =\frac{v_{n-1}}{v_{n}\left(u_{n} v_{n-1}-u_{n-1} v_{n}\right)}, \\
c_{i+1, i}=c_{i, i+1} & =\frac{1}{u_{i} v_{i+1}-u_{i+1} v_{i}}, \quad i=1, \ldots, n-1 .
\end{aligned}
$$

The following class of correlation matrices with important financial applications was introduced by Schoenmakers and Coffey (see [29]). A matrix $A=\left(a_{i j}\right)_{1 \leq i, j \leq n}$ is called a Schoenmakers-Coffey matrix associated to parameters $\left\{b_{i}\right\}_{1 \leq i \leq n}$ if

$$
a_{i j}:=\frac{\min \left\{b_{i}, b_{j}\right\}}{\max \left\{b_{i}, b_{j}\right\}}
$$

where

P0' $\left\{b_{i}\right\}_{1 \leq i \leq n}$ is positive,

$\mathrm{P} 1,\left\{b_{i}\right\}_{1 \leq i \leq n}$ is strictly increasing,

P2' $\left\{\frac{b_{i}}{b_{i+1}}\right\}_{1 \leq i \leq n-1}$ is strictly increasing, i.e. $\left\{b_{i}\right\}$ is strictly log-concave.

Taking $u_{i}:=b_{i}$ and $v_{i}:=\frac{1}{b_{i}}$ for all $i$, we can observe that a Schoenmakers-Coffey matrix is a Green matrix, with $g_{i j}=b_{i} / b_{j}$ when $i<j, g_{i j}=b_{j} / b_{i}$ when $i>j$ and $g_{i i}=1$ when $i=j$. Observe that P0', P1' and P2' imply properties P0, P1 and P2, respectively. Sometimes, in order to get a more flexible correlation structure, requiring P2 is not convenient. So, following [18], we can consider a slightly more general class of matrices $A=\left(a_{i j}\right)_{1 \leq i, j \leq n}$ (which we call extended SchoenmakersCoffey matrices associated to parameters $\left.\left\{b_{i}\right\}_{1 \leq i \leq n}\right)$ by requiring that $a_{i j}$ satisfies

$$
a_{i j}:=\operatorname{sign}\left(b_{i} b_{j}\right) \frac{\min \left\{\left|b_{i}\right|,\left|b_{j}\right|\right\}}{\max \left\{\left|b_{i}\right|,\left|b_{j}\right|\right\}}
$$

for all $i, j$ and, in addition: 
P1" $\left\{\left|b_{i}\right|\right\}_{1 \leq i \leq n}$ is strictly increasing,

that is, without requiring $\mathrm{P} 0$ ' and $\mathrm{P} 2$ ' and slightly weakening $\mathrm{P} 1$ '.

Finally, in order to consider positive matrices, we shall define an intermediate class of matrices between Schoenmakers-Coffey matrices and extended Schoenmakers-Coffey matrices given by the positive extended Schoenmakers-Coffey matrices, so that $A>0$ (i.e., $a_{i j}>0$ for all $i, j$ ). That is, $A=\left(a_{i j}\right)_{1 \leq i, j \leq n}$ is a positive extended Schoenmakers-Coffey matrix associated to parameters $\left\{b_{i}\right\}_{1 \leq i \leq n}$ if P1" holds, $a_{i j}$ satisfies (1) for all $i, j$ and the following property holds

P0" $\left\{b_{i}\right\}_{1 \leq i \leq n}$ with the same strict sign.

Observe that P0" and P1" imply that either $0<b_{1}<\cdots<b_{n}$ or $b_{n}<\cdots<b_{1}<0$ and then (1) becomes $a_{i j}=b_{i} / b_{j}$ when $i \leq j$. Hence a positive extended Schoenmakers-Coffey matrix is also a Green matrix. Taking into account also the symmetry of a Schoenmakers-Coffey matrix, we also have

$$
a_{i j}=\frac{b_{i}}{b_{j}}=\frac{b_{i} / b_{1}}{b_{j} / b_{1}}=\frac{1 / a_{1 i}}{1 / a_{1 j}} .
$$

A particular case of Schoenmakers-Coffey matrices is provided by the known Lehmer matrices $A=\left(a_{i j}\right)_{1 \leq i, j \leq n}$, which are defined by

$$
a_{i j}= \begin{cases}i / j, & \text { if } j \geq i \\ j / i, & \text { if } j<i .\end{cases}
$$

These matrices have been considered, for example, by N.J. Higham in [12] and [13]: the version 3.0 of the Test Matrix Toolbox for MATLAB 4.2 also includes Lehmer matrices. In the current versions of MATLAB “gallery('lehmer',n)" returns the Lehmer matrix of order $n$.

From now on, we shall denote by $\left\{u_{i}\right\}_{1 \leq i \leq n}$ the parameters associated to a positive extended Schoenmakers-Coffey matrix $A=\left(a_{i j}\right)_{1 \leq i, j \leq n}$. Then the $(i, j)$ entry of $A$ is given by

$$
a_{i j}=\frac{u_{i}}{u_{j}}, \quad i \leq j
$$

Let us observe that, by (2), given a positive extended Schoenmakers-Coffey matrix $A=$ $\left(a_{i j}\right)_{1 \leq i, j \leq n}$, it can be associated to the following particular parameters $\left\{u_{i}\right\}_{1 \leq i \leq n}$ given by

$$
u_{1}=1, \quad, u_{j}=\frac{1}{a_{1 j}}, \quad j=2, \ldots, n .
$$

In order to guarantee that positive extended Schoenmakers-Coffey matrices are positive definite, and so correlation matrices, we have to recall some concepts of Total Positivity theory. Le us recall that a matrix is totally positive if all its minors are nonnegative and it is strictly totally positive if they are positive (see [14, 23]).

The following result is derived from Corollary 3.8 of [1].

\section{Lemma 1}

A nonsingular totally positive matrix has all its principal minors positive. Therefore, a nonsingular symmetric totally positive matrix is positive definite.

A totally positive matrix $A$ is oscillatory if a certain power $A^{p}$ becomes strictly totally positive, and they are characterized (see Theorem 4.2 of [1]) in the following way.

\section{Proposition 1}

A matrix $A$ is oscillatory if and only if it is a nonsingular totally positive matrix with all its superdiagonal and subdiagonal entries are positive.

For nonsingular Green matrices, total positivity implies oscillatoriness, as the following result shows. 


\section{Proposition 2}

Let $G=\left(g_{i j}\right)_{1 \leq i, j \leq n}$ be a Green matrix associated to $\left\{u_{i}\right\}_{1 \leq i \leq n}$ and $\left\{v_{i}\right\}_{1 \leq i \leq n}$. Then the following properties are equivalent:

(i) $G$ is totally positive and nonsingular.

(ii) $\left\{u_{i}\right\}_{1 \leq i \leq n},\left\{v_{i}\right\}_{1 \leq i \leq n}$ have the same sign (i.e. $u_{i} v_{j}>0$, for all $1 \leq i, j \leq n$ ) and $\left\{u_{i} / v_{i}\right\}$ is strictly increasing.

(iii) $G$ is oscillatory.

Proof

(i) $\Longrightarrow$ (ii). By c) of p. 79 of [9], $G$ is totally positive if and only if

$$
\frac{u_{1}}{v_{1}} \leq \frac{u_{2}}{v_{2}} \leq \cdots \leq \frac{u_{n}}{v_{n}}
$$

and the rank of $G$ coincides with one plus the number of strict signs in (2.4). Therefore (i) implies (ii).

(ii) $\Longrightarrow$ (iii). Again by c) of p. 79 of [9], $G$ is totally positive and nonsingular. Since $g_{i, i+1}:=$ $u_{i} v_{i+1}=g_{i+1, i}$ for all $i=1,2, \ldots, n-1$, we deduce from the fact that $\left\{u_{i}\right\}_{1 \leq i \leq n},\left\{v_{i}\right\}_{1 \leq i \leq n}$ are nonzero with the same sign and from Proposition 1 that $G$ is oscillatory.

(iii) $\Longrightarrow$ (i). It holds because, by Proposition 1, an oscillatory matrix is totally positive and nonsingular.

The previous result characterizes Green matrices that are oscillatory or nonsingular totally positive matrices. Analogously, one could try to characterize Green matrices that are strictly totally positive matrices. But these properties are never simultaneously satisfied because, given a Green matrix $G$, we can find null minors for any $i, j$ with $i \leq j$ :

$$
\operatorname{det} G[i, i+1 \mid j, j+1]=u_{i} v_{j} u_{i+1} v_{i+1}-u_{i} v_{i+1} v_{j} u_{i+1}=0,
$$

which shows that $G$ is not strictly totally positive.

The following result is proved in Corollary 4 of of [18].

Theorem 2

A positive extended Schoenmakers-Coffey matrix is oscillatory.

Since a positive extended Schoenmakers-Coffey matrix is symmetric and, by Theorem 2 and Proposition 1 it is totally positive and nonsingular, we deduce from Lemma 1 that these matrices are positive definite and so correlation matrices. Another consequence of Theorem 2 is that all their eigenvalues are positive and different (see Theorem 6.5 of [1]) and that the number of variation of signs in the consecutive components of the eigenvectors corresponding to their $k$ th greater eigenvalue is $k-1, k=1, \ldots, n$. This last property for the cases $k=1,2,3$ corresponds to properties mentioned in Corollary 4 of [18] as level (also called shift in [25] and [26]), slope and curvature, respectively. These oscillations of the first three eigenvectors can give important information in financial problems (see [18, 25, 26, 27]).

We now introduce the last definitions of this section. Let us recall that a square matrix whose offdiagonal entries are all nonpositive is called a $Z$-matrix and that nonsingular $Z$-matrix $A$ is called an $M$-matrix if $A^{-1} \geq 0 . M$-matrices present important applications, including their application to the Leontief's input-output model, which is an outstanding example of the importance of mathematical modelling in economics (see [22], [2]).

By Theorem 2, a positive extended Schoenmakers-Coffey matrix is oscillatory and so its first three eigenvectors satisfy level, slope and curvature, as commented above. The following result presents several characterizations of these matrices, which are also correlation matrices satisfying $\mathrm{P} 0$ and P1. As a consequence, we also characterize nonnegative Green matrices that are nonsingular totally positive and have unit diagonal. 


\section{Theorem 3}

Let $G=\left(g_{i j}\right)_{1 \leq i, j \leq n}$ be a Green matrix with unit diagonal associated to $\left\{u_{i}\right\}_{1 \leq i \leq n},\left\{v_{i}\right\}_{1 \leq i \leq n}$. Then the following properties are equivalent:

(i) $G$ is totally positive and nonsingular.

(ii) $G$ is oscillatory.

(iii) $G$ is a positive extended Schoenmakers-Coffey matrix associated to parameters $\left\{u_{i}\right\}_{1 \leq i \leq n}$.

(iv) $G$ is a correlation matrix satisfying $\mathrm{P} 0$ and $\mathrm{P} 1$.

(v) $G^{-1}$ is a tridiagonal $M$-matrix

\section{Proof}

(i) $\Longrightarrow$ (ii). It is a consequence of Proposition 2 .

(ii) $\Longrightarrow$ (iii). If $G$ is oscillatory, then $G$ is totally positive and nonsingular by Proposition 1 . So, by Lemma 1, all principal minors of $G$ are positive and, since it is symmetric, it is positive definite. Therefore $G$ is a correlation matrix.

Since $G$ is a Green matrix, for each $i \leq j, g_{i j}=u_{i} v_{j}=g_{j i}$, where all $u_{i}, v_{j}$ are real numbers different from zero. Thus, $g_{i j} \neq 0$ for all $i \neq j$ and, since $G$ is oscillatory, $g_{i j}>0$ for all $i, j$. Since $g_{i i}=1$ for all $i=1, \ldots, n$, we also have that $v_{i}=1 / u_{i}$. Let us denote by $b_{i}:=u_{i}$ for all $i=1, \ldots, n$, and so $0<g_{i j}=b_{i} / b_{j}=g_{j i}$ for all $i \leq j$ and P0" holds. Observe that the sequence $\left\{u_{i} / v_{i}\right\}_{1 \leq i \leq n}$ becomes $\left\{b_{i}^{2}\right\}_{1 \leq i \leq n}$. By Proposition 2, $\left\{b_{i}^{2}\right\}_{1 \leq i \leq n}$ is strictly increasing and hence $\left\{\left|b_{i}\right|\right\}_{1 \leq i \leq n}$ is also strictly increasing and P1" and (iii) holds.

(iii) $\Longrightarrow$ (iv). It holds because, by Proposition 1, an oscillatory matrix is totally positive and nonsingular.

(iv) $\Longrightarrow$ (v). Since $G$ is a correlation matrix, it is positive definite symmetric, and so $G^{-1}$ is also positive definite symmetric. Since $G$ is a Green matrix, by Theorem $1, G^{-1}$ is tridiagonal, and its subdiagonal and superdiagonal entries entries have negative sign. In fact, using the notations of (ii) $\Longrightarrow$ (iii), Theorem 1 shows that that the $(i-1, i)$ entry of $G^{-1}(i=2, \ldots, n)$ is given by $\left(\left(b_{i} / b_{i+1}\right)-\left(b_{i+1} / b_{i}\right)\right)^{-1}$, which is negative because P0 and P1 imply that $\left\{b_{i}^{2}\right\}_{1 \leq i \leq n}$ is strictly increasing. In conclusion, $G^{-1}$ is a symmetric $Z$-matrix positive definite, and so it is a $Z$-matrix with all its principal minors positive. Then, by Theorem (2.3) of Chapter 6 of [3], it is a nonsingular $M$-matrix and (v) holds.

(v) $\Longrightarrow$ (i). If $G^{-1}$ is a tridiagonal $M$-matrix, then it is known (see Theorem 2.2 of [21]) that the nonsingular matrix $G$ is totally positive.

A matrix $A=\left(a_{i j}\right)_{1 \leq i, j \leq n}$ is called strictly diagonally dominant if $\left|a_{i i}\right|>\sum_{j \neq i}\left|a_{i j}\right|$, for each $i=1, \ldots, n$. Taking into account the well known fact that an strictly diagonally dominant $Z$-matrix is an $M$-matrix (cf. Theorem (2.3) of Chapter 6 of [2]), we can derive from Theorem 3 the following simple condition for a Green matrix $G$ to be a positive extended Schoenmakers-Coffey matrix: it is sufficient that $G^{-1}$ is a tridiagonal strictly diagonally dominant $Z$-matrix.

If we replace in Theorem 3 the hypothesis that $G=\left(g_{i j}\right)_{1 \leq i, j \leq n}$ has unit diagonal by its nonnegativity, then we can also derive an analogous characterization by using a decomposition of $G$, as the following result shows.

Corollary 1

Let $G=\left(g_{i j}\right)_{1 \leq i, j \leq n}$ be a nonnegative Green matrix. Then $G$ can be written as

$$
G=D C D
$$

where $D=\operatorname{diag}\left(\sqrt{g_{i i}}\right)_{i=1}^{n}$ and $C=\left(c_{i j}\right)_{1 \leq i, j \leq n}$ with $c_{i j}=\frac{g_{i j}}{\sqrt{g_{i i}} \sqrt{g_{j j}}}$ for all $i, j$. Moreover, the following properties are equivalent:

(i) $G$ is totally positive and nonsingular.

(ii) $G$ is oscillatory.

(iii) $C$ is a positive extended Schoenmakers-Coffey matrix.

(iv) $C$ is a correlation matrix satisfying $\mathrm{P} 0$ and $\mathrm{P} 1$. 
(v) $G^{-1}$ is a tridiagonal $M$-matrix

\section{Proof}

Observe that, if $G$ is a Green matrix, then it is associated to nonzero numbers $\left\{u_{i}\right\}_{1 \leq i \leq n},\left\{v_{i}\right\}_{1 \leq i \leq n}$ and $g_{i i}=u_{i} v_{i} \neq 0$ for all $i$. Since $G$ is nonnegative, $g_{i i}>0$ for all $i$ and we can write (6) with $D$ a positive diagonal matrix (i.e., with positive diagonal entries). Taking into account that nonsingularity, total positivity, oscillatoriness, tridiagonality or being a nonsingular $M$-matrix are preserved under multiplication by positive diagonal matrices, the equivalences of (i), (ii), (iii), (iv) and (v) follow from Theorem 3.

\section{BIDIAGONAL DECOMPOSITION OF THE CONVERSION OF POSITIVE EXTENDED SCHOENMAKERS-COFFEY MATRICES}

As shown in Theorem 3, a positive extended Schoenmakers-Coffey matrix $A$ is nonsingular totally positive. Then it satisfies a unique bidiagonal decomposition, denoted by $\mathcal{B D}(A)$ in [16]. In this last paper, assuming that the parameters of $\mathcal{B D}(A)$ are known with high relative accuracy (HRA), Koev presented algorithms for computing the eigenvalues, singular values and the inverse of the matrix $A$ with HRA, as well as the solution of linear systems $A x=b$ with the components of $b$ satisfying a chessboard pattern of alternating signs with HRA. In [17] we can find a software library, called TNTool, containing an implementation of the previous algorithms for Matlab and Octave. The corresponding functions are called TNEigenvalues, TNSingularValues, TNSolve and TNJInverse. They require as input argument the parameters of the bidiagonal decomposition $\mathcal{B D}(A)$ of the matrix $A$. The function TNSolve also needs a second argument, the vector of independent coefficients $b$ of the linear system $A x=b$ to be solved. Observe that TNJInverse provides the bidiagonal decomposition of $C=J A^{-1} J$, where $J=\operatorname{diag}\left(\left((-1)^{i}\right)_{i=0}^{n-1}\right)$, and so we compute $A^{-1}=J C J$ to HRA. In conclusion, if we get the bidiagonal decomposition of a positive extended Schoenmakers-Coffey matrix to HRA, then we would solve the previous algebraic problems to HRA using the software library of [17].

In this section, we provide a fast algorithm of $\mathcal{O}(n)$ elementary operations to derive the bidiagonal decomposition of the conversion of an $n \times n$ positive extended Schoenmakers-Coffey matrix $A$ to HRA, assuming that we know the parameters $\left\{u_{i}\right\}_{1 \leq i \leq n}$ associated to the matrix $A$ to HRA.

Let us first recall the mentioned result on the bidiagonal decomposition of a nonsingular totally positive matrix $A$ (see Theorem 4.1 of [11]).

\section{Theorem 4}

Let $A$ be a nonsingular $n \times n$ totally positive matrix. Then $A$ admits a decomposition of the form

$$
A=F_{n-1} \cdots F_{1} D G_{1} \cdots G_{n-1},
$$

where $F_{i}$ and $G_{i}, i \in\{1, \ldots, n-1\}$, are the lower and upper triangular nonnegative bidiagonal matrices given by

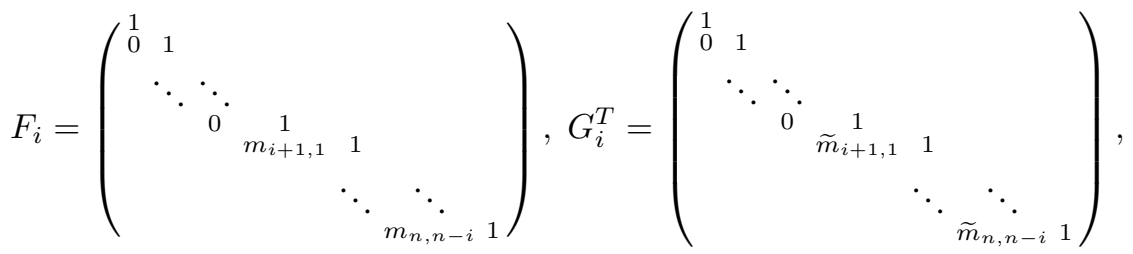

and $D$ a diagonal matrix $\operatorname{diag}\left(p_{11}, \ldots, p_{n n}\right)$ with positive diagonal entries. If, in addition, the entries $m_{i j}, \widetilde{m}_{i j}$ satisfy

$$
m_{i j}=0 \Rightarrow m_{h j} \quad \forall h>i
$$

and

$$
\widetilde{m}_{i j}=0 \Rightarrow m_{i k} \quad \forall k>j
$$

then the decomposition (7) is unique. 
Following [16], we will denote the unique bidiagonal decomposition (7) of a matrix $A$ by $\mathcal{B D}(A)$. In Theorem 4.1 of [11] it was also shown that $m_{i j}, \widetilde{m}_{i j}$, and $p_{i i}$ are the multipliers and the diagonal pivots of an elimination procedure called Neville elimination. Roughly speaking, Neville elimination is a procedure to create zeros in a matrix by means of adding to a given row a suitable multiple of the previous one (see [10]).

The following result provides the $L D L^{T}$-factorization of the inverse of a positive extended Schoenmakers-Coffey matrix, which will be used later to derive the mentioned bidiagonal decomposition of a positive extended Schoenmakers-Coffey matrix.

\section{Theorem 5}

Let $G$ be the $n \times n$ positive extended Schoenmakers-Coffey matrix associated to $\left(u_{i}\right)_{1 \leq i \leq n}$. Then its inverse $G^{-1}$ can be decomposed as:

$$
G^{-1}=E D^{-1} E^{T}
$$

where

$$
E=\left(\begin{array}{cccccccc}
1 & & & & & & & \\
f_{21} & 1 & & & & & & \\
& \ddots & \ddots & & & & & \\
& & \ddots & 1 & & & & \\
& & & f_{i, i-1} & 1 & & & \\
& & & & f_{i+1, i} & 1 & & \\
& & & & & \ddots & \ddots & \\
& & & & & & f_{n, n-1} & 1
\end{array}\right)
$$

and $D^{-1}=\operatorname{diag}\left(g_{11}, \ldots, g_{n n}\right)$, with

$$
\begin{aligned}
g_{i i} & =u_{i+1}^{2} \frac{1}{\left(u_{i+1}-u_{i}\right)\left(u_{i+1}+u_{i}\right)}(>0), \\
f_{i+1, i} & =-\frac{u_{i}}{u_{i+1}}(<0),
\end{aligned}
$$

for $i=1, \ldots, n-1$, and $g_{n n}=1$.

\section{Proof}

By Theorem 3, $G^{-1}$ is a tridiagonal nonsingular $M$-matrix and so it has positive leading principal minors (cf. Theorem (2.3) of Chapter 6 of [2]). Therefore we can perform its Gaussian elimination without row or column exchanges and it has an $L D U$-factorization, whose lower triangular matrix with unit diagonal is denoted by $E$ and whose diagonal matrix is denoted by $D^{-1}$. We will also denote by $f_{i j}$ the multipliers and by $g_{i i}$ the diagonal pivots of the Gaussian elimination of the matrix $G^{-1}$. Then, taking into account that $G^{-1}$ is symmetric and the uniqueness of the $L D U$-factorization we have that the upper triangular matrix with unit diagonal of the $L D U$-factorization of $G^{-1}$ is given by $E^{T}$. Now we only have to determine the multipliers $f_{21}, \ldots, f_{n, n-1}$ and the pivots $g_{i i}$. Gaussian elimination of the tridiagonal matrix $C=G^{-1}$ consists of $n-1$ successive steps, resulting in a sequence of matrices $C^{(1)}=C, C^{(2)} \ldots, C^{(n)}$. The matrix $C^{(i+1)}$ is obtained from $C^{(i)}$ making zero the entry $(i+1, i)$ by adding an adequate multiple of the $i$ th row to the $(i+1)$ th row. So let us prove that the pivots and multipliers of the procedure are given by (10) and (11), respectively, by induction on $i=1, \ldots, n-1$. For $i=1$, first we have by Theorem 1 and taking into account by (3) that $v_{i}=1 / u_{i}$, that

$$
g_{11}=c_{11}^{(1)}=\frac{u_{2}}{u_{1}\left(\frac{u_{2}}{u_{1}}-\frac{u_{1}}{u_{2}}\right)}=u_{2}^{2} \frac{1}{\left(u_{2}-u_{1}\right)\left(u_{2}+u_{1}\right)},
$$

that is, formula (10) for $i=1$. Second, applying a first step of Gaussian elimination we have

$$
f_{21}=\frac{c_{21}^{(1)}}{c_{11}^{(1)}}=\frac{c_{21}^{(1)}}{g_{11}}=\frac{\frac{1}{\frac{u_{1}}{u_{2}}-\frac{u_{2}}{u_{1}}}}{u_{2}^{2} \frac{1}{\left(u_{2}-u_{1}\right)\left(u_{2}+u_{1}\right)}}=-\frac{u_{1}}{u_{2}},
$$


that is, formula (11) for $i=1$. Now let us assume that formulas (10) and (11) hold for some $k \in\{1, \ldots, n-2\}$ and then let us prove them for $k+1$. We have by the tridiagonal structure of $G^{-1}$ that

$$
g_{k+1, k+1}=c_{k+1, k+1}^{(k+1)}=c_{k+1, k+1}^{(k)}-f_{k+1, k} c_{k, k+1}^{(k)}=c_{k+1, k+1}^{(1)}-f_{k+1, k} c_{k, k+1}^{(1)} .
$$

By the induction hypothesis and Theorem 1 we have

$$
\begin{aligned}
g_{k+1, k+1} & =\frac{\frac{u_{k+2}}{u_{k}}-\frac{u_{k}}{u_{k+2}}}{\left(\frac{u_{k+1}}{u_{k}}-\frac{u_{k}}{u_{k+1}}\right)\left(\frac{u_{k+2}}{u_{k+1}}-\frac{u_{k+1}}{u_{k+2}}\right)}+\frac{u_{k}}{u_{k+1}} \frac{1}{\frac{u_{k}}{u_{k+1}}-\frac{u_{k+1}}{u_{k}}} \\
& =u_{k+2}^{2} \frac{1}{\left(u_{k+2}-u_{k+1}\right)\left(u_{k+2}+u_{k+1}\right)},
\end{aligned}
$$

that is, formula (10) for $i=k+1$. Now, applying the $(k+1)$ th step of Gaussian elimination, we get

$$
f_{k+2, k+1}=\frac{c_{k+2, k+1}^{(k+1)}}{c_{k+1, k+1}^{(k+1)}}=\frac{c_{k+2, k+1}^{(1)}}{g_{k+1, k+1}}=\frac{\frac{1}{\frac{u_{k+1}}{u_{k+2}}-\frac{u_{k+2}}{u_{k+1}}}}{u_{k+2}^{2} \frac{1}{\left(u_{k+2}-u_{k+1}\right)\left(u_{k+2}+u_{k+1}\right)}}=-\frac{u_{k+1}}{u_{k+2}},
$$

that is, formula (11) for $i=k+1$ and the induction holds. Finally, we have for the last pivot of Gaussian elimination

$$
g_{n n}=c_{n n}^{(n)}=c_{n n}^{(n-1)}-f_{n, n-1} c_{n-1, n}^{(n-1)}=c_{n n}^{(1)}-f_{n, n-1} c_{n-1, n}^{(1)} .
$$

By Theorem 1 and formula (11) for $i=n-1$, we have

$$
g_{n n}=\frac{\frac{1}{u_{n-1}}}{\frac{1}{u_{n}}\left(\frac{u_{n}}{u_{n-1}}-\frac{u_{n-1}}{u_{n}}\right)}+\frac{u_{n-1}}{u_{n}} \frac{1}{\left(\frac{u_{n-1}}{u_{n}}-\frac{u_{n}}{u_{n-1}}\right)}=1 .
$$

Finally, the signs in (10) and (11) follow from P0" and P1".

Given a matrix $A=\left(a_{i j}\right)_{1 \leq i, j \leq n}$, we define the conversion matrix of $A$ as $A^{\#}=\left(a_{i j}^{\#}\right)_{1 \leq i, j \leq n}:=$ $\left(a_{n+1-i, n+1-j}\right)_{1 \leq i, j \leq n}$, which can be written as $A^{\#}=P A P$ where $P$ is obtained from the identity matrix by reversing the order of its rows. Since $G=P G^{\#} P$, let us analyze the corresponding algebraic problems mentioned above for $G$ in terms of $G^{\#}$. Observe that, since positive extended Schoenmakers-Coffey matrices are symmetric, their singular values coincide with their eigenvalues.

- Eigenvalues of $G$. Since $P^{-1}=P$, the eigenvalues of the matrix $G$ coincide with those of $G^{\#}$. So, using the bidiagonal decomposition of $G^{\#}$ to HRA and the function TNEigenvalues of TNTool, we can derive the eigenvalues of $G$ with HRA.

- Solution of a system of linear equations $G x=b$ such that $b$ has a chessboard sign pattern. Taking into account that $b=G x=P G^{\#} P x$ and that $P^{-1}=P$, we conclude that the system is equivalent to $G^{\#} y=P b$ where $y=P x$. Observe that, if $b$ has a chessboard sign pattern, then $P b$ also has a chessboard sign pattern. Then, using the bidiagonal decomposition $\mathcal{B D}\left(G^{\#}\right)$ to HRA with the function TNSolve, we get $y=P x$ to HRA. So, from $P y$, which only reverses the order of the entries of $y$, we get $x$ to HRA.

- Inverse of the matrix $G, G^{-1}$. By $P^{-1}=P$ and $G=P G^{\#} P$, we derive $G^{-1}=P\left(G^{\#}\right)^{-1} P$. So, using $\mathcal{B D}\left(G^{\#}\right)$ to HRA with the functionTNJ Inverse we get $\mathcal{B D}\left(J\left(G^{\#}\right)^{-1} J\right)$ to HRA. Thus, with the function TNExpand of [17], this last bidiagonal decomposition and the usual matrix product we derive $G^{-1}$ to HRA.

In conclusion, if we obtain the bidiagonal decomposition of $G^{\#}$ to HRA we will be able to solve the previous algebraic problems to HRA for $G$.

We define the matrix $E_{i}(\alpha)$ as the $n \times n$ matrix differing from the identity matrix only in its $(i, i-1)$ entry, which is $\alpha$ instead of 0 . 


\section{Theorem 6}

Let $G$ be the $n \times n$ positive extended Schoenmakers-Coffey matrix associated to $\left(u_{i}\right)_{1 \leq i \leq n}$. Then, $G^{\#}$ can be decomposed as:

$$
G^{\#}=F_{n-1} F_{n-2} \cdot F_{1} D F_{1}^{T} \cdots F_{n-2}^{T} F_{n-1}^{T},
$$

where $F_{i}:=E_{i+1}\left(m_{i+1,1}\right)$ for $i=1, \ldots, n-1$ and $D=\operatorname{diag}\left(p_{11}, \ldots, p_{n n}\right)$, with

$$
\begin{aligned}
p_{n+1-i, n+1-i} & =\frac{\left(u_{i+1}-u_{i}\right)\left(u_{i+1}+u_{i}\right)}{u_{i+1}^{2}}\left(=\frac{1}{g_{i i}}>0\right), \\
m_{i+1,1} & =\frac{u_{n-i}}{u_{n+1-i}}\left(=-f_{n+1-i, n-i}>0\right),
\end{aligned}
$$

for $i=1, \ldots, n-1$, and $p_{11}=1$. Moreover, if the parameters $\left(u_{i}\right)_{1 \leq i \leq n}$ are known to HRA, then the computation of (13) and (14) (and so, of the $\mathcal{B D}\left(G^{\#}\right)$ given by (12)) can be performed to HRA and with $2 n-1$ quotients and $n-1$ additions, subtractions and products.

\section{Proof}

By Theorem 5, $G^{-1}=E D^{-1} E^{T}$ with $E=E_{2}\left(f_{21}\right) E_{3}\left(f_{32}\right) \cdots E_{n}\left(f_{n, n-1}\right)$ and $D^{-1}=$ $\operatorname{diag}\left(g_{11}, \ldots, g_{n n}\right)$, where the $f_{i+1, i}$ 's and the $g_{i i}$ 's are defined by (10) and (11). Then we derive

$$
\begin{aligned}
G & =\left(E^{-1}\right)^{T} D E^{-1} \\
& =E_{2}^{T}\left(-f_{21}\right) E_{3}^{T}\left(-f_{32}\right) \cdots E_{n}^{T}\left(-f_{n, n-1}\right) D E_{n}\left(-f_{n, n-1}\right) \cdots E_{3}\left(-f_{32}\right) E_{2}\left(-f_{21}\right) .
\end{aligned}
$$

Then, taking the conversion in the previous expression

$$
\begin{aligned}
G^{\#} & =\left(E_{2}^{T}\left(-f_{21}\right)\right)^{\#} \cdots\left(E_{n}^{T}\left(-f_{n, n-1}\right)\right)^{\#} D^{\#}\left(E_{n}\left(-f_{n, n-1}\right)\right)^{\#} \cdots\left(E_{2}\left(-f_{21}\right)\right)^{\#} \\
& =E_{n}\left(-f_{21}\right) E_{n-1}\left(-f_{32}\right) \cdots E_{2}\left(-f_{n, n-1}\right) D^{\#} E_{2}^{T}\left(-f_{n, n-1}\right) \cdots E_{n-1}^{T}\left(-f_{32}\right) E_{n}^{T}\left(-f_{21}\right) .
\end{aligned}
$$

Denoting $m_{i+1,1}:=-f_{n+1-i, n-i}=\frac{u_{n-i}}{u_{n+1-i}}$ and $F_{i}:=E_{i+1}\left(m_{i+1,1}\right)$ for $i=1, \ldots, n-1$, (12) holds and, by Theorem 4, it gives $\mathcal{B D}\left(G^{\#}\right)$. Finally, (13) and (14) can be computed from the sequence $\left(u_{i}\right)_{1 \leq i \leq n}$ to HRA because the only subtractions use the initial parameters of the sequence and their signs are given by (10) and (11).

Observe that the previous procedure to derive

$$
\mathcal{B D}\left(A^{\#}\right)
$$

to HRA has been provided assuming that we know the parameters $\left(u_{i}\right)_{1 \leq i \leq n}$ associated to the positive extended Schoenmakers-Coffey matrix $A$ to HRA. Under this assumption, we can also know $A$ to HRA by (3) because it only uses quotients. Conversely, if we know $A$ to HRA, then we can also obtain associated parameters $\left(u_{i}\right)_{1 \leq i \leq n}$ to HRA by (4), and so we can perform the previous mentioned algebraic problems for $A$ to HRA.

\section{NUMERICAL TESTS}

By using the algorithms mentioned in the previous section, the eigenvalues (which coincide with the singular values), the inverses and the solutions of some linear systems with positive extended Schoenmakers-Coffey matrices can be computed to HRA. Now we will illustrate the results presented in Section 3 with numerical examples. In our numerical tests we have considered the positive extended Schoenmakers-Coffey matrices of orders $n=100,200, \ldots, 1000$ associated to the parameters $(\sqrt{i})_{i=1}^{n}$, which will be denoted by $G_{100}, G_{200}, \ldots, G_{1000}$, respectively. 


\subsection{Eigenvalues}

First we have computed the eigenvalues of $G_{100}, \ldots, G_{1000}$ using Mathematica with a 100 digits precision. In addition, we have also computed approximations to the eigenvalues of those matrices with the usual Matlab command eig and with TNEigenvalues of software library [17] using the bidiagonal decomposition to HRA of $\left(G_{n}\right)^{\#}, n=100, \ldots, 1000$, given in Theorem 6 . Then we have taken the approximations of the lowest eigenvalue of each of the matrices and we have computed the corresponding relative errors considering eigenvalues obtained by Mathematica as the exact eigenvalues.

In Figure 1, we can see the relative errors of the approximation to the lowest eigenvalue of each matrix. We can observe that the bidiagonal decomposition to HRA of the conversion of $\left(G_{100}\right)^{\#}, \ldots,\left(G_{1000}\right)^{\#}$ joint with Plamen Koev software library provide more accurate results than these obtained by using eig Matlab command.

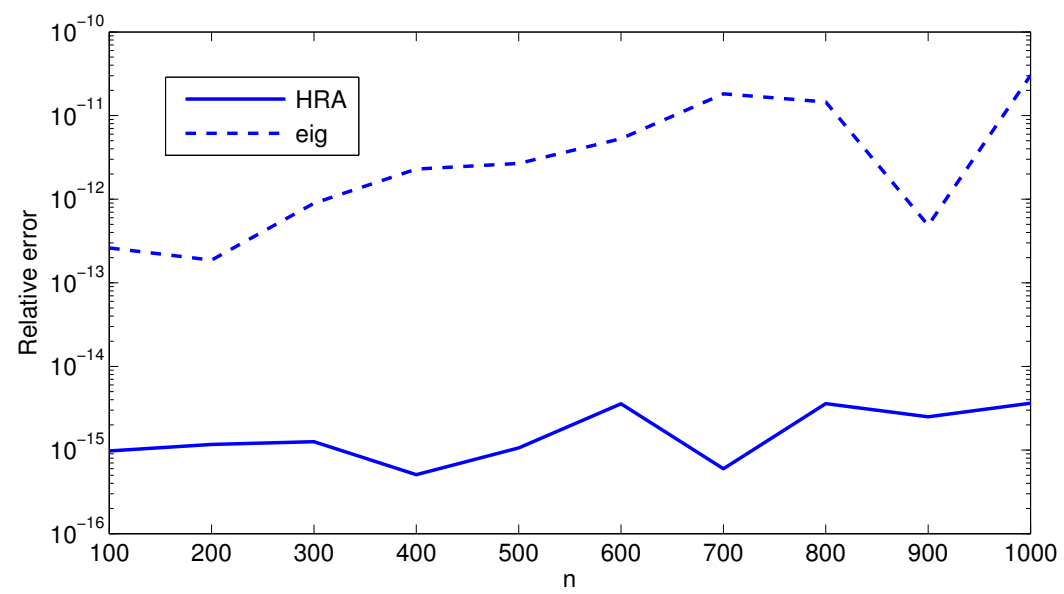

Figure 1. Relative errors when computing the lowest eigenvalue of $G_{100}, G_{200}, \ldots, G_{1000}$

\subsection{Inverse of positive extended Schoenmakers-Coffey matrices}

We have considered again $G_{100}, G_{200}, \ldots, G_{1000}$. We have computed its inverse using Mathematica with 100 digits precision. In addition, we have also computed approximations to the inverse with the usual Matlab command inv and with TNJInverse using the bidiagonal decomposition of its converses to HRA given in Theorem 6. We have calculated the componentwise relative errors for both approximations considering the inverse obtained with Mathematica as exact. The relative error for the entry with the greatest error can be seen in Table I.

\begin{tabular}{|r|c|}
\hline$n$ & HRA \\
\hline 100 & $2.7970963 \mathrm{e}-14$ \\
200 & $3.6618939 \mathrm{e}-14$ \\
300 & $9.8424379 \mathrm{e}-14$ \\
400 & $1.2602160 \mathrm{e}-13$ \\
500 & $1.4459874 \mathrm{e}-13$ \\
600 & $1.5218884 \mathrm{e}-13$ \\
700 & $1.5563051 \mathrm{e}-13$ \\
800 & $1.6575193 \mathrm{e}-13$ \\
900 & $1.9978553 \mathrm{e}-13$ \\
1000 & $2.1002671 \mathrm{e}-13$ \\
\hline
\end{tabular}

Table I. Maximum relative error when computing the inverse of $G_{n}$ 
As we know, the inverse of a positive extended Schoenmakers-Coffey is a tridiagonal matrix, but when this inverse is computed by the usual inv Matlab command, the approximation of the inverse obtained have all its entries different from zero. So, componentwise relative errors cannot even be computed.

\subsection{Solution of a linear system of equations}

We have considered the system of linear equations

$$
G_{1000} x=b,
$$

where $b$ has a alternating pattern sign and the absolute value of each of its components have been randomly generated as an intenger in the interval $[1,1000]$. Then we have computed an approximation to the solution with Mathematica using 100 digits precision, and approximations to this one with Matlab command \and with TNSolve of the software library [17] using the bidiagonal factorization of $G_{1000}^{\#}$ to HRA given in Theorem 6. Then we have computed the relative errors componentwise, its means an the maximum relative error. Table II shows the results obtained. We can observe again that HRA methods outperform usual Matlab commands. This advantage joins with the very low computational cost of the bidiagonal decomposition of the conversion of the positive extended Schoenmakers-Coffey, as shown in Theorem 6.

\begin{tabular}{|r|c|c|}
\hline & Maximal relative error & Mean relative error \\
\hline HRA & $1.0834303 \mathrm{e}-13$ & $2.3728132 \mathrm{e}-14$ \\
Matlab command $\backslash$ & $2.8056129 \mathrm{e}-10$ & $7.9525457 \mathrm{e}-12$ \\
\hline \multicolumn{2}{|c|}{ Table II. Relative errors when solving system of linear equations $G_{1000} x=b$}
\end{tabular}

\section{ACKNOWLEDGEMENT}

This work was partially supported by the Spanish Research grant MTM2015-65433-P (MINECO/FEDER), Gobierno de Aragón, and Fondo Social Europeo

\section{REFERENCES}

1. Ando, T. (1987) Totally positive matrices, Linear Algebra Appl. 90, pp. 165-219.

2. Berman, A. and Plemmons, R. J. (1979) Nonnegative matrices in the mathematical sciences (Computer Science and Applied Mathematics. Academic Press, New York-London).

3. Barrett, W. W. (1979) A theorem on inverse of tridiagonal matrices, Linear Algebra Appl. 27, pp. 211-217.

4. Capovani M. (1970) Sulla determinazione della inversa delle matrici tridiagonali e tridiagonali a blocchi, Calcolo 7, pp. 295-303.

5. Carnicer, J. M., Peña, J. M. and Pinkus, A. (2002) On zero-preserving linear transformations, J. Math. Anal. Appl. 266 , pp. 237-258.

6. J. Delgado, J. M. Peña, Accurate computations with collocation matrices of rational bases, Appl. Math. Comput. 219 (2013) 4354-4364.

7. J. Delgado, J. M. Peña, Accurate computations with collocation matrices of q-Bernstein polynomials, SIAM J. Matrix Anal. Appl. 36 (2015) 880-893.

8. J. Demmel, M. Gu, S. Eisenstat, I. Slapnicar, K. Veselic and Z. Drmac, Computing the singular value decomposition with high relative accuracy, Linear Algebra Appl. 299 (1999) 21-80.

9. Gantmacher, F. P. and Krein M. G. (2002) Oscillation matrices and kernels and small vibrations of mechanical systems (Revised edition. AMS Chelsea Publishing, Providence, RI).

10. M. Gasca and Peña J. M. (1992) Total positivity and Neville Elimination, Linear Algebra Appl. 165, pp. $25-44$.

11. M. Gasca, J. M. Peña, On factorizations of totally positive matrices. In: Total Positivity and Its Applications (M. Gasca and C.A. Micchelli, Ed.), Kluver Academic Publishers, Dordrecht, The Netherlands (1996), 109-130.

12. N.J. Higham, Algorithm 694: A collection of test matrices in MATLAB, ACM Trans. Math. Software 17 (1991), 289-305.

13. N.J. Higham, The Test Matrix Toolbox for Matlab (Version 3.0), Manchester Centre for Computational Mathematics, Numerical Analysis Report No. 276, September 1995.

14. Karlin S. (1968) Total Positivity. Vol.1 (Stanford University Press, Stanford CA).

15. P. Koev, Accurate eigenvalues and SVDs of totally nonnegative matrices, SIAM J. Matrix Anal. Appl. 21 (2005) $1-23$. 
16. P. Koev, Accurate computations with totally nonnegative matrices, SIAM J. Matrix Anal. Appl. 29 (2007) 731-751.

17. P. Koev, http://math.mit. edu/ plamen/software/TNTool.html

18. Lord, R. and Pelsser, A. (2007) Level, Slope and Curvature: Art or Artefact?, Appl. Math. Finance 14, pp. 105-130.

19. A. Marco, J. J. Martínez, A fast and accurate algorithm for solving Bernstein-Vandermonde linear systems, Linear Algebra Appl. 422 (2007) 616-628.

20. A. Marco, J. J. Martínez, Accurate computations with totally positives Bernstein-Vandermonde matrices, Electronic Journal of Linear Algebra 26 (2013), 357-380.

21. Peña, J. M. (1995) M-matrices whose inverses are totally positive, Linear Algebra Appl. 221, pp. 189-193.

22. Peña, J. M. (2008) Hierarchical open Leontief models, Linear Algebra Appl. 428, pp. 2549-2559.

23. Pinkus, A. (2010) Totally Positive Matrices (Cambridge University Press, Cambridge).

24. Rebonato, R. (2002) Modern Pricing of Interest-Rate Derivatives (Princeton University Press, Princeton).

25. Salinelli, E. and Sgarra, C. (2006) Correlation matrices of yields and total positivity, Linear Algebra Appl. 418, pp. 682-692.

26. Salinelli, E. and Sgarra, C. (2007) Shift, slope and curvature for a class of yields correlation matrices, Linear Algebra Appl. 426, pp. 650-666.

27. Salinelli, E. and Sgarra, C. (2011) Some results on correlation matrices for interest rates, Acta Appl. Math. 115, pp. 291-318.

28. Salinelli, E., Serra-Capizzano and Sesana D. Eigenvalue-eigenvector structure of Schoenmakers-Coffey matrices via Toeplitz technology and applications. To appear in Linear Algebra Appl. (D. O.I.: 10.1016/j.laa.2015.03.017).

29. Schoenmakers, J. and Coffey, B. (2003) Systematic Generation of Parametric Correlation Structures for the LIBOR Market Model, Int. J. Theor. Appl. Finance 6, pp. 507-519. 